\title{
The 3D CFD Study of Gliding Swimmer on Passive Hydrodynamics Drag
}

\author{
Vishveshwar Rajendra Mantha ${ }^{1,2,3^{*}}$, Daniel Almeida Marinho ${ }^{2,4}$, Antonio Jose Silva ${ }^{2,3}$ and \\ Abel Ilah Rouboa ${ }^{1,5}$ \\ ${ }^{1}$ Department of Mechanical Engineering; University of Trás-os-Montes and Alto Douro; Vila Real - Portugal. \\ ${ }^{2}$ Centre of Research in Sports; Health and Human Development; CIDESD; Vila Real - Portugal. ${ }^{3}$ Department of \\ Sport Sciences; Exercise and Health; University of Trás-os-Montes and Alto Douro; Vila Real - Portugal. \\ ${ }^{4}$ Department of Sport Sciences; University of Beira Interior; Covilhã - Portugal. ${ }^{5}$ Department of Mechanical \\ Engineering and Applied Mechanics; University of Pennsylvania; Philadelphia - USA
}

\begin{abstract}
The aim of this study was to analyze the effect of depth on the hydrodynamic drag coefficient during the passive underwater gliding after the starts and turns. The swimmer hydrodynamics performance was studied by the application of computational fluid dynamics (CFD) method. The steady-state CFD simulations were performed by the application of $k$ - omega turbulent model and volume of fluid method to obtain two-phase flow around a threedimensional swimmer model when gliding near water surface and at different depths from the water surface. The simulations were conducted for four different swimming pool size, each with different depth, i.e., 1.0, 1.5, 2.0 and $3.0 \mathrm{~m}$ for three different velocities, i.e., $1.5,2.0$ and $2.5 \mathrm{~m} / \mathrm{s}$, with swimmer gliding at different depths with intervals of $0.25 \mathrm{~m}$, each starting from the water surface, respectively. The numerical results of pressure drag and total coefficients at individual average race velocities were obtained. The results showed that the drag coefficient decreased as depth increased, with a trend toward reduced fluctuation after $0.5 \mathrm{~m}$ depth from the water surface. The selection of the appropriate depth during the gliding phase should be a main concern of swimmers and coaches.
\end{abstract}

Key words: Computational Fluid Dynamics (CFD), Passive Drag, Starts, Turns, Streamline Gliding Position, Glide depth

\section{INTRODUCTION}

The swimming race is an event of modern Olympics, in which milliseconds can make a difference between the gold and silver. Extremely small winning margins still justify the incorporation of possible improvements in strength, style, conditioning, etc. The swimmers performance is decided by the athletes' physical active strength, sport technique and resulting hydrodynamic performance (Hanna 2006).
In the swimming event, the total swim time consists of the sum of three parts: starting time, swim time and turn times (Guimarães and Hay 1985). The underwater glide after the starts and turns assumes a high influence on the outcome of a swimming event (Vilas-Boas et al. 2010). Thus, the study of existing fluid flow around the swimmer appears to be important so that there is an improvement in the performance. In the events, which have many turns, some authors (Vilas-Boas et al. 2000; Cossor and Mason 2001) suggest that, apart from the influence of starting position

*Author for correspondence: vishveshwar@gmail.com 
adopted by a swimmer, the body alignment and path chosen during the glide phase also contribute towards determining the success in the event. To enable better performance, the swimmer should minimize the hydrodynamic drag (not being the exception, the phases of starting and turning), adopting a position as hydrodynamic as possible (Barbosa et al. 2006). In an aquatic environment, three types of hydrodynamic drag forces, which oppose the movement of the swimmer, are i) friction drag, ii) form or pressure drag, and iii) wave drag (Toussaint et al. 2002; Polidori et al. 2006).

A way of minimizing the hydrodynamic drag is to increase the depth of the underwater slide, since the contribution of wave drag seems to decrease when the swimmers perform their glide at higher depths (Lyttle et al. 2000; Polidori et al. 2006; Marinho et al. 2009). The hydrodynamics study of the glide movement in the passive underwater position has been carried out using the experimental methods (Clarys and Jiskoot 1975; Kolmogorov and Duplishcheva, 1992; Lyttle et al. 1999; Vilas-Boas et al. 2010). More recently, it was concluded that, to a depth less than $0.7 \mathrm{~m}$, drag produced could be 2.4 times higher than for deeper than $0.7 \mathrm{~m}$; also that the wave drag produced was responsible for $50-60 \%$ of the total drag (Vennell et al. 2006). However, there is need to study this, not only because there are few studies conducted, but because of the variability in results obtained, along with the difficulties involved in performing these experimental protocols (Bixler et al. 2007).

In the investigative studies of this type, the application of CFD method in swimming could be an alternative to the experimental studies (Bixler et al. 1996). The possibility of obtaining data, without performing the experimental tests using human beings, is a huge asset not only in terms of the costs, but also in terms of time efficiency, since, at any time, there is ability to make a new simulation and change the variables, allowing the comparative studies. In this sense, this work aimed to investigate the effect of depth in the hydrodynamic drag of a swimmer during the underwater glide, using CFD method. The hypothesis of the current study was that the hydrodynamic drag decreased along with the reduction in the fluctuation with increasing depth of the gliding swimmer. There would be a range of optimum depth, which could be chosen by the swimmer during the glide.

\section{MATERIALS AND METHODS}

To carry out the numerical simulation studies, the geometry under consideration could be studied to calculate and deduce the parameters required for the analysis. The fluid flow can be completely simulated by solving Navier-Stokes equations, but this requires expensive computational resources. To save on the economics, these equations are transformed into algebraic form and solved by solver algorithms on the finite discretized domain consisting of volumetric mesh with the prediction of fluctuating velocities with the help of turbulent model. The air-water twophase fluid flow is estimated by the VOF model.

The steady state numerical simulations were performed for the velocity of 1.5, 2.0 and $2.5 \mathrm{~m} / \mathrm{s}$. The simulation of swimmer gliding with steady velocity was implemented by keeping the swimmer static in the fluid flowing at constant velocity. The flow around the swimmer was turbulent, corresponding to the Reynolds number varying between the order of 5 to 6 (Bixler et al. 2007). Due to that reason, the Reynolds averaged Navier-Stokes equations with the Boussinesq hypothesis to model the Reynolds stresses (Hinze 1975) were considered. The closure problem of the turbulent modeling was solved using $\mathrm{k}$ $\omega$ model. The advantage of the $k-\omega$ model over the $k-\varepsilon$ model was its improved performance for the boundary layers under adverse pressure gradients and it could be applied throughout the boundary layer, including the viscous-dominated region, without further modification (Wilcox 1998). The detailed terms of the $\mathrm{k}-\omega$ model transport equations used in the present study are provided in user manual of Fluent documentation (Fluent 2006).

In reality, the swimmer moved through two fluid, i.e., air (which was in gas phase) and water (which was in liquid phase) when gliding at water surface and also there was displacement of surface water when swimmer was gliding below the surface, leading to energy losses. The twophase simulations by implementation of volume of fluid (VOF) method simulated the actual physics of the problem with flow of air-water as seen in pool environment. Most of the previous studies have not simulated two-phase flow around the swimmer while studying the passive glide.

The simulations were based on the finite volume method of discretization (Patankar 1980). In order to limit the numerical dissipation, particularly 
when the geometry was complex consisting of an unstructured grid, as seen in Figure 1, with the choice of second order upwind discretization scheme for the convection terms in the solution equations, and Pressure-Implicit with Splitting of Operators (PISO) pressure-velocity coupling scheme for the double precision, pressure-based solver was chosen. The PISO pressure-velocity coupling scheme, part of the SIMPLE family of algorithms, was based on the higher degree of the approximate relation between the corrections for the pressure and velocity (Fluent 2006). The convergence criterion chosen was equal to $10^{-6}$.

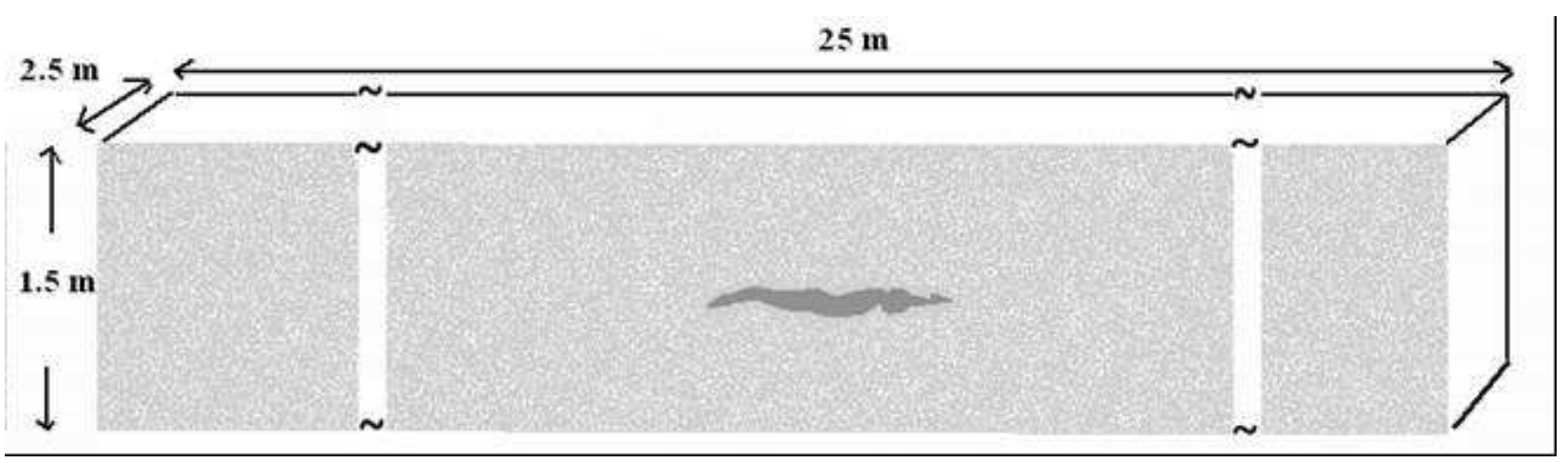

Figure 1 - The representation of the typical overall geometric domain of computation with tetrahedral mesh volume with swimmer model in glide position.

The 3D surface geometry model was acquired through the standard commercial L.A.S.E.R. scanner, which had an average maximal error circumference of less than $1.0 \mathrm{~mm}$, with point cloud density 27 points $/ \mathrm{cm}^{2}$. The swimmer who served as the basis for obtaining a digital model was $2.40 \mathrm{~m}$ tall in streamline glide position, with a perimeter of the head, chest, waist and hip of 0.58 , $1.02,0.87$ and $0.93 \mathrm{~m}$, respectively. The 3D surface geometry data of swimmer model was generated in Solidworks ${ }^{(\mathrm{R})}$ CAD software (Fig.1) and exported in IGES format for import into GAMBIT preprocessor. The grid structure and the computational domain are shown in Figure 1. The upstream boundary was located at two swimmer height from the tip of fingers. The downstream extent was located more than six times swimmer height from the tip of toe fingers. In the fluid domain, swimmer was positioned proportionately near to the inlet, instead of middle. By doing so, flow in the back of the swimmer could be resolved more accurately, and also limited the front region that had no practical interest, since the fluid was mostly unperturbed by the swimmer before it touched the fingers. The appropriate boundary conditions were applied on the computational domain, with wall boundary condition on swimmer surface and bottom surface of computational domain, symmetric boundary condition on top, rear and front surfaces of computational domain, velocity inlet and pressure outlet on the remaining side surfaces of computational domain, respectively. The simulation of swimmer glide motion with steady velocity was implemented by keeping the swimmer static in the fluid flowing at constant velocity. The study of drag was simplified by not considering the effect of surface wind waves, generally present on the swimming pool water surface, since their contribution to drag was presumed to be less predominant.

\section{RESULTS AND DISCUSSION}

In swimming, the total drag is composed of the friction drag, pressure drag and wave drag. When the swimmer glided immediately below the water surface, the displacement of water surface around the swimmer occurred, which generated the waves (Fig. 2A). The water waves around the swimmer clearly showed variation, as water flew over the swimmer's body. Swimming near the surface of the water caused the formation of waves on the surface, resulting in the so-called drag effect of the wave. For the swimmer gliding at the surface, the wave drag component was not calculated in this 
study due to inherent limitation of CFD software's available, but the estimated drag was considered as component of the total drag, which excluded the contribution from wave drag.

The pressure drag was caused by the pressure differential between the front and the rear the swimmer, which was proportional to the square of swimming speed, the density of water and frontal cross-sectional area of the swimmer. The contours of static pressure showed variation over the body and the value increased with increase in depth (Fig. 2B). The simulation of two-phases, i.e., air and water as seen in the pool environment was important to predict the drag forces realistically. When the pressure drag (pressure force) variation from all the four swimming pools was studied at different velocities, different glide depths in respective swimming pools, it indicated a rise initially, followed by brief calm down with less amount of variation and fluctuation in the later stage (Fig. 3) with similar tendency observed by variation of hydrodynamic drag coefficient (Fig.
4). In general, for all the speeds, the hydrodynamic drag decreased with increasing depth and there was a tendency to stabilize (with less amount of variation) the coefficient of drag between $0.5 \mathrm{~m}$ to $0.15 \mathrm{~m}$, but the range varied depending upon the depth of swimming pool. The lower value of drag when the swimmer was just below the surface of water, could be attributed to the drag contributed only by the pressure effects and entirely from the swimmers body exposed by the water and remaining prominent contribution was from the wave drag, which was not estimated in the current study. The drag experienced by the swimmer must be more when gliding near the air-water interface and the wave component will decrease thereafter as swimmer glides deeper, giving rise to prominence of pressure drag (Toussaint et al. 2002; Vennell et al. 2006). Naturally, the increase in swimmer glide velocity, will witness augmentation in all the components of drag depending upon respective location of swimmer during glide.

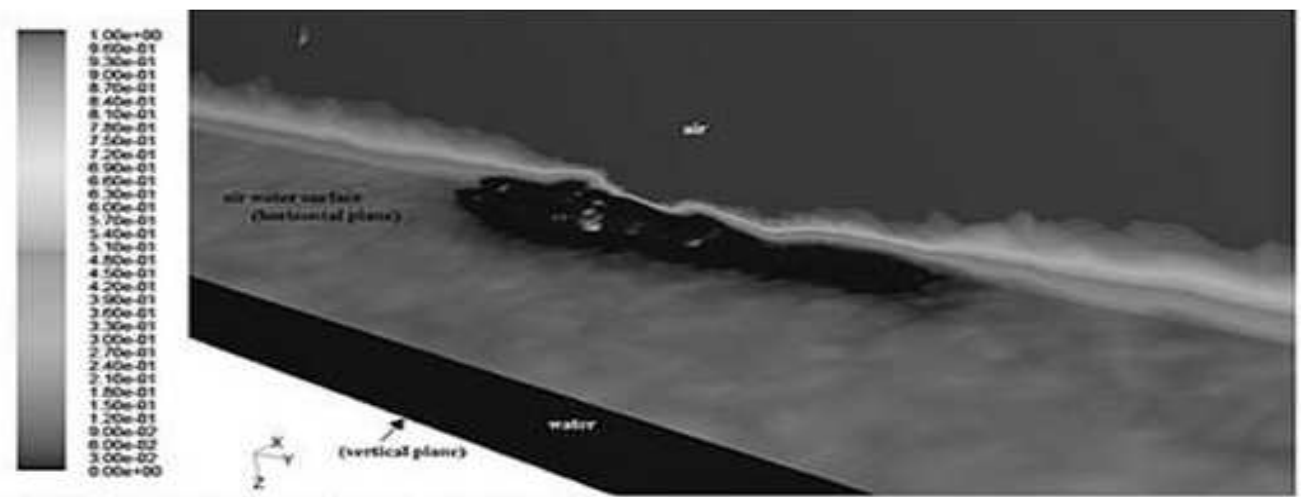

A. Contours of volume fraction (water-air)

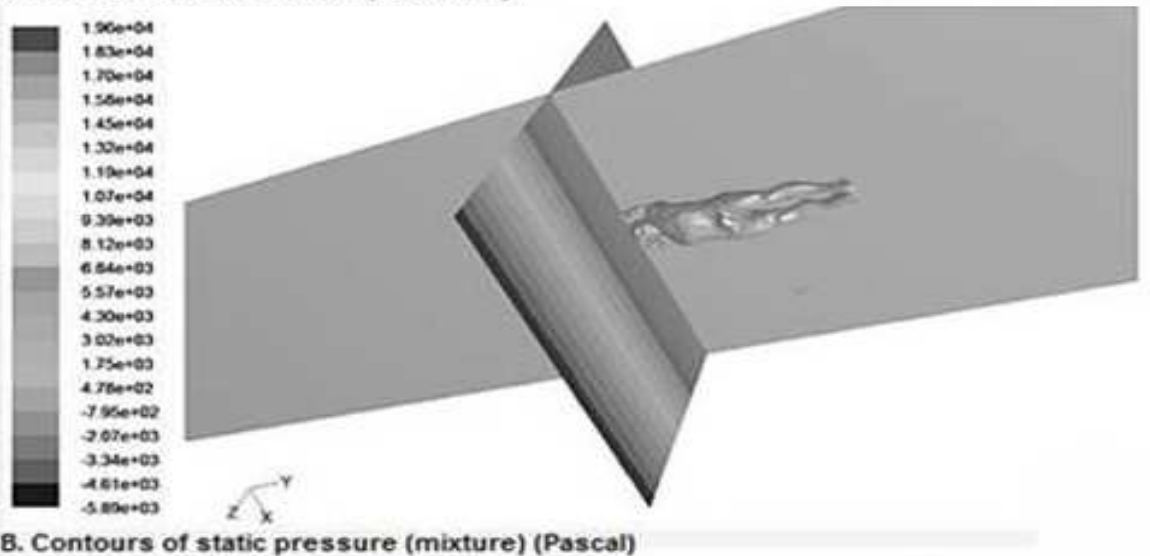

Figure 2 - (A) The contours of volume fraction of air (phase-1) around swimmer model on horizontal plane at air water interface and on vertical plane, (B) The contours of static pressure (Pa) around swimmer and its variation with depth plotted on vertical plane at average flow velocity of $2.5 \mathrm{~m} / \mathrm{s}$. 

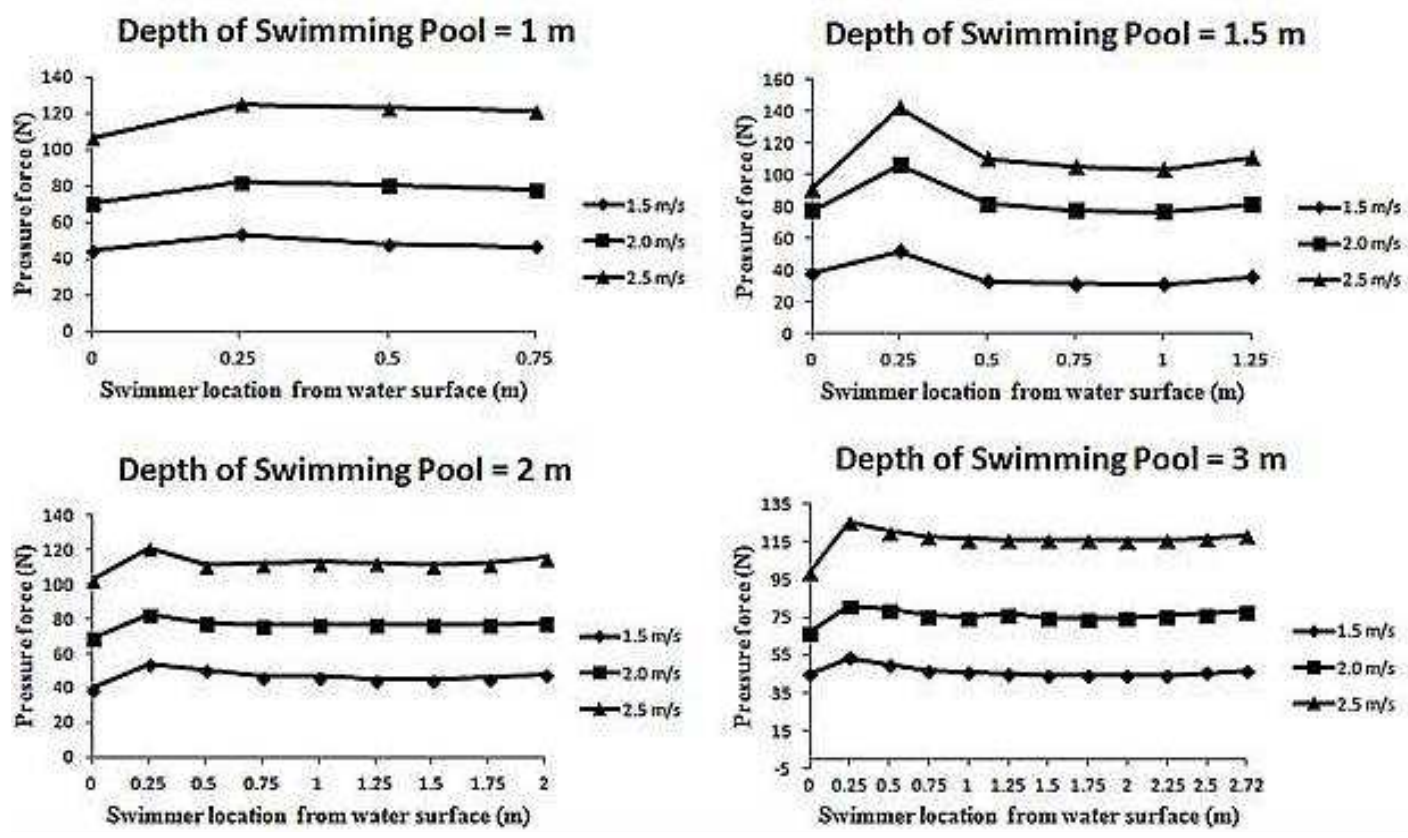

Figure 3 - The variation of pressure force for different average velocities and various glide depths for four different swimming pools.

Depth of Swimming Pool $=1 \mathrm{~m}$

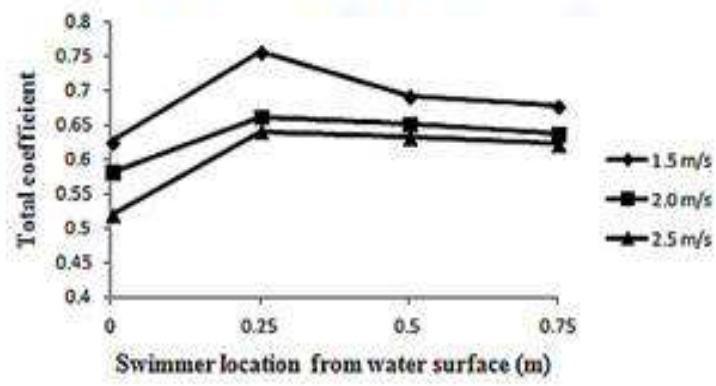

Depth of Simming Pool $=2 \mathrm{~m}$

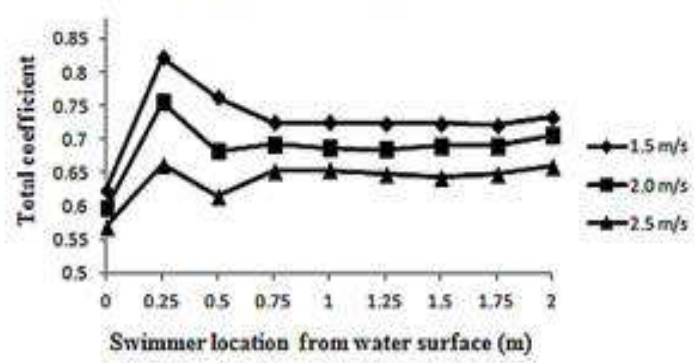

Depth of Swimming Pool $=1.5 \mathrm{~m}$

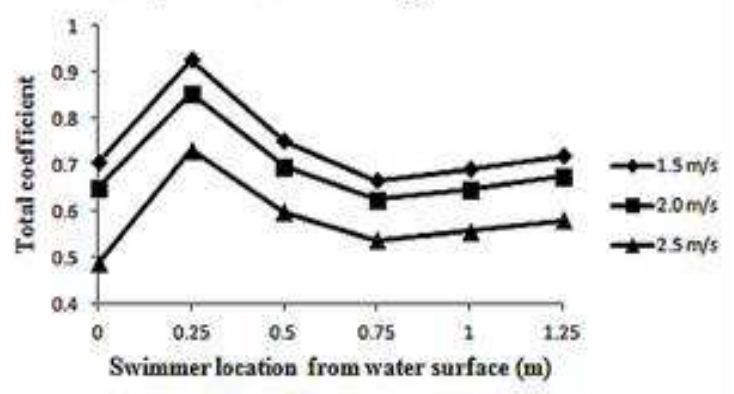

Depth of Swimming Pool $=3 \mathrm{~m}$

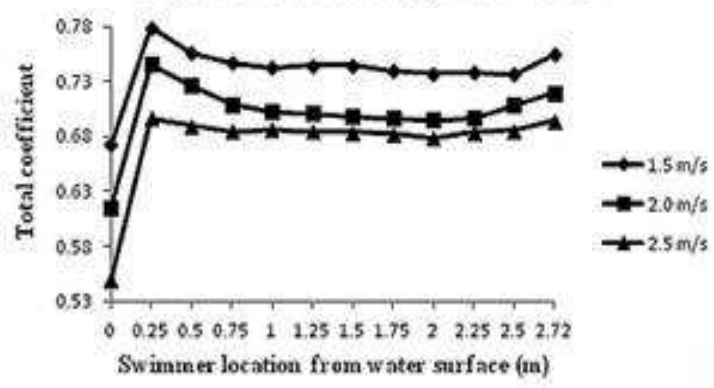

Figure 4 - The variation of total coefficient for different average velocities and various glide depths for four different swimming pools.

The main objective of this study was to evaluate the effect of swimmer glide depth on the hydrodynamic drag and its coefficient by using CFD method. It is noteworthy that with increase in the depth of underwater glide, the hydrodynamic drag decreased and became stable (with reduced amount of variation) in a range depending upon the depth of the swimming pool. The location of 
the stabilized region was where the contribution from the surface waves was also minimal (Lyttle et al. 1999; Toussaint et al. 2002; Vennell et al. 2006). This could be of practical application to choose the sustainable optimal glide path depending upon the glide velocity and depth of the swimming pool. The current study showed that the hydrodynamic drag coefficient decreased as the depth increased, which was in agreement with previous studies (Lyttle et al. 1999; Vennell et al. 2006; Bixler et al. 2007; Marinho et al. 2009).

When the swimmer glided at $1.0 \mathrm{~m}$ glide depth in a $1.5 \mathrm{~m}$ deep swimming pool at the speed of 2.5 $\mathrm{m} / \mathrm{s}$, the numerical study predicted $18 \%$ more drag as compared with the experimental measurements (Vennell et al. 2006). This could be attributed to the variation and differences in the experimental and numerical conditions, such as the characteristics of swimmers, depth of swimming pool, differences in streamline glide positions, fluctuations in actual glide depth during the swimming, and combined error in the measurement instruments and numerical errors (Bixler et al. 2007). This could also be attributed to the differences in the position of hands, due to procedures followed during the scanning process in obtaining three-dimensional model, location of the model's hands (side by side and slightly apart), which also contributed to higher values of overall drag values (Vorontsov and Rumyantsev 2000). The differences between the previous numerical studies could be also attributed to the limitation in previous studies with the lack of use of real 3D swimmer model and limitations on the simulation of actual physics as compared with the present study, which was complete in this aspect, leading to more accurate prediction.

The values of the friction drag were noticeably similar. This was as expected, as the friction drag was produced depending upon the amount of exterior surface area of swimmer's body in contact with water; this value would not suffer large oscillations, since the model used was the same and the swimmer was fully submerged, except when gliding near the water surface (Vennell et al. 2006). For glide depths in proximity to the water surface, the swimmers body was submerged partly and it depended upon the velocity and irregularity in formation and dissipation of water waves. This introduced deviation in the estimation of wetted surface area and also submerged frontal cross section area of the swimmers body, which in turn produced fluctuations in drag. However, it would be essential to quantify the wave drag numerically, which in the present study was not analyzed due to limitations presented by the numerical code.

With reference to the variation of drag coefficient with glide depth, there was a brief initial affinity for fluctuation and reduction in drag for all the three glide velocities at different glide depths in the four types of swimming pools studied. There was also a marked tendency to retain the comparable value of drag between $0.5 \mathrm{~m}$ to 1.75 $\mathrm{m}$, depending upon the depth of swimming pool under the study. Some of the previous studies indicated that after $0.6 \mathrm{~m}$ of glide depth from water surface, the wave drag was almost negligible, and it did not contribute significantly towards total drag (Lyttle et al. 1999). It is noteworthy that with increase in the depth of underwater glide, the hydrodynamic drag decreased and became almost unwavering, which could be identified as a stabilized region (with less amount of variation) depending upon the depth of the swimming pool. The location of the stabilized region was at a depth, where the contribution from the surface waves was predicted to be minimal (Toussaint et al. 2002; Vennell et al. 2006). Although, wave drag was not calculated due to inherent limitations, this did not affect the aim and outcome of the present study, as swimmers are practically instructed to glide deep, especially after the starts and turns, and this was seen to be implemented in practice. This study could be of practical application to the swimmers, helping them choose the sustainable optimal glide path, glide depth depending upon the glide velocity and depth of the swimming pool.

\section{CONCLUSION}

The drag decreased as the depth increased and there was a tendency for stabilization of the value, which varied for the glide velocities and depth of the swimming pool. With increase in the depth of underwater glide, the hydrodynamic drag decreased and became almost stable, which was identified as stabilized region depending upon the depth of the swimming pool. This could be of practical application to the swimmers, helping them choose the sustainable optimal glide path, glide depth depending upon the glide velocity and depth of the swimming pool. Thus, the selection of the appropriate depth for the underwater glide should be a vital concern of the swimmers and 
coaches. However, the ideal depth must be one that allows to reduce the hydrodynamic drag as much as possible, but at the same time, allowing a optimum vertical distance from the water surface, so that the swimming could be restarted. The balance of the drag reducing (increasing depth) and increasing the vertical distance traveled are the key aspects to consider. The current study presented the knowledge of hydrodynamics, which could be utilized by an athlete or a coach during the progression at different race velocities in different swimming pool environments.

\section{ACKNOWLEDGMENTS}

This work was supported by a grant of the Science and Technology Foundation Portugal (PTDC/DES/098532/2008).

\section{REFERENCES}

Barbosa TM, Keskinen K, Vilas-Boas JP. Bioenergetic and biomechanical factors limiting the yield of pure swimming sports. Movement. 2006; 2: 201-13.

Bixler B, Pease D, Fairhurst F. The accuracy of computational fluid dynamics analysis of the passive drag of a male swimmer. Sports Biomech. 2007; 6: 81-98.

Bixler BS, Schloder M. Computational fluid dynamics: An analytical tool for the 21 st century swimming scientist. Swim J Res. 1996; 11: 4-22.

Clarys JP, Jiskoot J. Total resistance of selected body positions in the front crawl. In: Clarys JP, Lewillie L, editors. Swimming II. Baltimore, USA: University Park Press; 1975. 110-117.

Cossor J, Mason B. Swim start performances at the Sydney 2000 Olympic Games. In: XIX Swim Sessions of the Symposium on in Sports Biomechanics: Proceedings Contributed papersabstracts; 2001; San Francisco, USA: University of San Francisco; 2001; 1: 70-74.

FLUENT 6.3 Documentation. [Internet]. 2011 Nov 1 [updated 2012 Jan 1; cited 2013 Jan 1.]. Available from:www.jullio.pe.kr/fluent6.1/help/html/ug/main_p re.htm.

Guimaraes A, Hay J. The mechanical analysis of the grab starting technique in swimming. Int J Sports Biomech. 1985; 1: 25-35.

Hanna RK. Going Faster, Higher and Longer in Sport with CFD. In: Haake S, editor. The Engineering of Sport. Rotterdam, Netherlands: Taylor and Francis Press; 2006. p. 3-10.

Hinze JO. Turbulence. 1st ed. New York, USA: McGraw-Hill Publishing; 1975.
Kolmogorov S, Duplishcheva O. Active drag, useful mechanical power output and hydrodynamic force coefficient in different swimming strokes at maximal velocity. J Biomech. 1992; 25: 311-18.

Lyttle A, Blanksby B, Elliot B, Lloyd D. Net Forces During tethered simulation of underwater streamlined gliding and kicking technique of the freestyle turn. $J$ Sports Med Sci. 2000; 18: 801-7.

Lyttle A, Blansksby B, Elliott B, Lloyd D. Optimal depth for streamlined gliding. In: Keskinen KL, Komi PV, Hollander AP, editors. In: Biomechanics and medicine in swimming VIII. Jyvaskyla: Gummerus Printing. 1999. p. 165-70.

Marinho DA, Barbosa TM, Reis VM, Kjendlie PL, Alves FB, Vilas-Boas J et al. Swimming propulsion forces are enhanced by the small finger spread. J Appl Biomech. 2010; 26: 87-92.

Marinho DA, Reis V, Alves F, Vilas-Boas J, Machado L, Silva A, et al. During gliding Hydrodynamic drag in swimming. J Appl Biomech. 2009; 25: 253-57.

Patankar SV. Numerical Heat Transfer and Fluid Flow. 1 st ed. Washington DC, USA: Hemisphere Publishing Corporation. 1980.

Polidori G, Taiar R, Fohanno S, Mai T, Lodini A. Skinfriction drag analysis from the forced convection modeling in simplified underwater swimming. $J$ Biomech. 2006; 39: 2535-2541.

Toussaint H, Truijens M, Elzinga M-J, De Ven A, De Best H, Snabel B. Effect of a fast-skin ${ }^{\mathrm{TM}}$ 'body' suit on drag During front crawl swimming. Sports Biomech. 2002; 1: 1-10.

Vennell R, Pease D, Wilson B. Wave drag on human swimmers. J Biomech. 2006; 39 (4): 664-671.

Vilas-Boas J, Costa L, Fernandes R, Ribeiro J, Figueiredo P, Marinho D. Determination of the drag coefficient During the first and second positions of the gliding underwater breaststroke stroke. J Appl. Biomech. 2010; 26: 324-331.

Vilas-Boas JP, Cross MJ., Sousa F. Conceição F, Carvalho JM. Integrated kinematic and dynamic analysis of two-track start techniques. In: R. Sanders R, Hong Y, editors. In: Proceedings of the International Symposium on XVIII in Sports Biomechanics, Applied Program - Application of Biomechanical Study in Swimming. Hong Kong, China: The Chinese University Press. 2000. p. 11317.

Vorontsov AR, Rumyantsev VA. Resistive forces in swimming. In: Zatsiorsky VM, editors. Biomechanics in Sport: Performance enhancement and injury prevention. Oxford, USA: Blackwell. 2000. p. 184204.

Wilcox, D. C. Turbulence Modeling for CFD. 1st ed. La Canada, California, USA: DCW Industries Press. 1998.

Received: August 05, 2013; Accepted: December 23, 2013. 